\title{
Avaliação de Acidentes de Trabalho com Materiais Biológicos em Médicos Residentes, Acadêmicos e estagiários de um Hospital-Escola de Porto Alegre
}

\author{
Evaluation of Occupational Accidents with \\ Biological Materials in Medical Residents, \\ Academics and Interns of School Hospital of \\ Porto Alegre
}

\author{
Rafael Teixeira de Souzal \\ Claudia Giuliano Bica \\ Carmen Susana Mondadori ${ }^{I I}$ \\ Alana Durayski Ranzi
}

PALAVRAS-CHAVE:

- Acidentes de trabalho.

- Estudantes.

- Saúde pública.

- Accidents, Occupational.

- Students.

- Public health.

Recebido em: 16/09/2011

Reencaminhado em: 02/01/2012

Aprovado em: 06/02/2012

REVISTA BRASILEIRA DE EDUCAÇÃO MÉDICA 118 36(1):118-124; 2012

I Universidade Federal de Ciências da Saúde de Porto Alegre, Porto Alegre, RS, Brasil.

"I Santa Casa de Misericórdia de Porto Alegre, Porto Alegre, RS, Brasil. 


\section{INTRODUÇÃO}

Historicamente, os profissionais de saúde não eram considerados como categoria profissional de alto risco para acidentes ocupacionais. A preocupação com riscos biológicos surgiu a partir da constatação dos agravos à saúde dos profissionais que exerciam atividades em laboratórios nos quais ocorria a manipulação com microrganismos e material clínico desde o início da década de 40. Para profissionais que atuam na área clínica, entretanto, somente a partir da epidemia da AIDS (Síndrome da Imunodeficiência Adquirida) nos anos 80 é que as normas para as questões de segurança no ambiente de trabalho passaram a ser amplamente adotadas ${ }^{1}$.

Acidentes ocupacionais são fenômenos socialmente determinados, indicativos da intensa rotina de trabalho à qual é submetida grande parte dos trabalhadores de saúde. Constituem importante problema de saúde pública no Brasil, atingindo principalmente adultos jovens e causando elevado número de casos de invalidez permanente e, inclusive, óbitos ${ }^{2,3,4}$.

A exposição ocupacional por material biológico é entendida como a possibilidade de contato com sangue e fluidos orgânicos no ambiente de trabalho, e as formas de exposição incluem inoculação percutânea, por intermédio de agulhas ou objetos cortantes, e o contato direto com pele e/ou mucosas 5 .

A grande preocupação com os riscos ocupacionais a que estão sujeitos os trabalhadores de saúde está relacionada ao desempenho de suas funções, uma vez que eles manipulam materiais biológicos e sofrem acidentes, o que pode provocar sérios agravos à saúde. São várias as circunstâncias que geram condições para a ocorrência de acidentes com materiais biológicos na área da saúde, principalmente o ambiente físico de trabalho, o que pode repercutir tanto na saúde do trabalhador quanto em prejuízos para a empresa ou instituição.

O ambiente de trabalho hospitalar é considerado insalubre, por agrupar indivíduos portadores de diversas enfermidades infecto-contagiosas, que se destacam como as principais fontes de transmissão de microrganismos patogênicos ${ }^{6}$. Os hospitais, além disso, viabilizam muitos procedimentos que oferecem riscos de acidentes para os trabalhadores da saúde com contato direto com fluidos corpóreos durante a realização de procedimentos invasivos ou através da manipulação de artigos, roupas, lixo e até mesmo as superfícies contaminadas ${ }^{6,7}$.

Existem dois componentes básicos envolvidos em acidentes ocupacionais: o fator humano e o ambiental. $\mathrm{O}$ fator humano pode atuar mediante a predisposição individual para a ocorrência de problemas físicos, psíquicos, familiares, profissionais e socioculturais. $\mathrm{O}$ fator ambiental é evidenciado quando o trabalhador é exposto a determinados agentes externos que, ao se manifestarem, causam o evento mórbido, culminando por incrementar os dados epidemiológicos de acidentes ocupacionais com materiais biológicos ${ }^{6}$.

Em um estudo, Guimarães (2004) mostra que a relação entre incidência geral de acidentes ocupacionais e morbidade não é tão baixa, evidenciando um pequeno potencial de mortalidade e um grande potencial de morbidez, condição que demanda estratégias para minimizar esses agravos no ambiente de trabalho. Contudo, a despeito dos esforços despendidos para minimizar os acidentes ocupacionais, ainda são preocupantes os registros de acidentes de trabalho e doenças profissionais no Brasil ${ }^{8}$.

O presente estudo objetiva realizar um levantamento de dados sobre acidentes ocupacionais com materiais biológicos, através da revisão das "Comunicações de Acidentes de Trabalho" e planilhas de atendimento no "Serviço de Atenção à Saúde e Qualidade de Vida - SASQV", responsável pelo atendimento dos acidentados na instituição, no período de janeiro de 2007 a julho de 2009. O estudo envolve médicos residentes, acadêmicos e estagiários das áreas da saúde, os quais frequentaram um hospital-escola da cidade de Porto Alegre.

\section{MATERIAIS E MÉTODOS}

Trata-se de um estudo transversal, histórico, descritivo, de vigilância epidemiológica de acidentes ocupacionais.

A população estudada foi constituída por médicos residentes, acadêmicos e estagiários de um hospital-escola, os quais realizaram notificação de acidente com material biológico no período de janeiro de 2007 a julho de 2009, no serviço médico do hospital.

O instrumento de coleta de dados foi a "Ficha de Comunicação de Acidente de Trabalho", idêntica ao modelo disponibilizado pelo Ministério da Saúde para notificação dos acidentes com materiais biológicos, e ela se encontra arquivada no órgão responsável pela notificação dos acidentes na instituição.

Foi feita uma análise descritiva dos dados obtidos; as variáveis quantitativas foram apresentadas como médias, e as variáveis qualitativas como percentual. Nas análises bivariadas foi utilizado o teste de Pearson (Qui-Quadrado), considerando como significativo um $\mathrm{p}<0,05$.

Este estudo de pesquisa foi aprovado sob $\mathrm{n}^{\mathrm{o}}$ de parecer 09/473, pelo CEP — UFCSPA, estando de acordo com a Resolução 196/96.

\section{RESULTADOS}

De janeiro de 2007 a julho de 2009 foram notificados no "Serviço de Atenção à Saúde e Qualidade de Vida — SASQV" 166 acidentes de trabalho - envolvendo médicos residentes, acadêmicos e estagiários - ocorridos no hospital-escola. A tota- 
lidade dos acidentes ocupacionais foi registrada como acidentes típicos, não havendo nenhum acidente relatado como de trajeto.

Do total de sujeitos que frequentaram o hospital no período de 2007 a 2009 (7445 sujeitos), constatamos que 2,23\% destes se acidentaram. Ao investigarmos a frequência absoluta de acidentados por categoria profissional realizando uma correlação com o total de sujeitos que trabalharam no hospital-escola, constatamos que havia, no período do estudo, ${ }^{26}$ "Acadêmicos de Biomedicina", com 7,69\% de acidentados; 705 "Médicos Residentes", com 6,81\% acidentados; 1070 "Acadêmicos de Medicina", com 4,11\% de acidentados; 2875 "Estagiários Técnicos de Enfermagem", com 1,84\% de acidentados; 1399 "Acadêmicos de Enfermagem", com 0,71\% de acidentados; e 1370 "Acadêmicos de Fisioterapia", com 0,66\% de acidentados.

Ao observarmos o contingente de acadêmicos acidentados levando em consideração a categoria profissional em relação com o número total de acidentes, percebemos que os "Estagiários Técnicos de Enfermagem" são os que mais relataram acidentes (31,93\%), seguidos dos "Médicos Residentes" (28,92\%), "Acadêmicos de Medicina" (26,51\%), "Acadêmicos de Enfermagem" (6,02\%), "Acadêmicos de Fisioterapia" (5,42\%), e dos "Acadêmicos de Biomedicina" (1,20\%) (Tabela 1).

As variáveis investigadas no estudo foram as seguintes: 1) porcentagem de acidentes por categoria; 2) sexo; 3) faixa etária; 4) trimestre em que ocorreu o acidente; 5) sítio anatômico envolvido; e 6) tipo de lesão. Todas essas variáveis são apresentadas na Tabela 1.

Nesta mesma análise, quando discriminamos o tipo de material ou o meio causador do acidente com material biológico, verificamos que 59,64\% ocorreram com agulhas, seguidos de $12,65 \%$ com secreções e $9,04 \%$ com sangue (Figura 1).

A frequência de acidentes ocupacionais correlacionados ao status sorológico dos pacientes fontes envolvidos demonstra que $7,23 \%$ e $18,67 \%$ dos acidentes envolveram paciente fonte reagente para HIV e HCV respectivamente.

Considerando a causa do acidente, 60,24\% relataram que o motivo foi o descuido do próprio sujeito de pesquisa; 7,83\% relataram reencape de agulhas; $7,23 \%$, realização do HGT (hemoglicoteste); 6,63\%, descuido de terceiros; 5,42\% relataram não haver disponibilidade de equipamento de proteção individual adequado; $3,61 \%$ relataram que o motivo do acidente foi a agitação do paciente; e 1,56\% destacou haver descarte de material em local inadequado (Figura 2).

Não foi evidenciada diferença estatística em nenhum dos testes utilizados para comparação, tanto das variáveis qualitativas, como das quantitativas.

\section{DISCUSSÃO}

Neste estudo, avaliamos os tipos e causas mais frequentes de acidentes envolvendo material biológico nas categorias acadêmicas envolvidas, determinando a frequência com que estes ocorreram e suas características peculiares.

Das categorias profissionais analisadas, a dos "Estagiários Técnicos de Enfermagem", "Médicos Residentes" e "Acadêmicos de Medicina" foram as mais envolvidas em acidentes com materiais biológicos, totalizando 87,36\% dos 166 acidentes de trabalho no período do estudo.

Diversos autores [(Pinho et al. (2007), Nishide et al. (2004) e Marcus et al. (1999)] investigaram a frequência de acidentes com materiais biológicos e evidenciaram que a equipe de enfermagem é responsável por 30 a 50\% em média dos relatos de acidentes em hospitais ${ }^{9,10,11}$.

Podemos evidenciar, em nosso estudo, que, dentro da equipe de enfermagem, os "Estagiários Técnicos de Enfermagem" tiveram a maior frequência de relatos de acidentes, pois eles estão mais expostos a fatores de risco devido à sua permanência na maior parte do tempo em assistência direta aos pacientes e à execução de vários procedimentos invasivos, sendo os materiais pérfuro-cortantes um dos seus principais instrumentos de trabalho na prática diária. Desta forma, os dados de acidentes evidenciados durante o período de formação desses profissionais se traduzem posteriormente na prática profissional.

Os "Médicos Residentes", conforme nosso estudo, foram responsáveis pelo segundo lugar em relatos de acidentes, corroborando, assim, os estudos realizados por Ciorlia et al. (2004) e Caixeta et al. (2005) ${ }^{12,6}$. Eles evidenciaram que a equipe médica possui grande envolvimento nos acidentes ocupacionais, principalmente os que envolvem materiais pérfuro-cortantes. Isso acontece porque os "Médicos Residentes" estão na linha de frente do atendimento ao paciente e realizam, frequentemente, a coleta de sangue para exames de urgência e procedimentos de maior complexidade, ou seja, aqueles que não são delegáveis aos alunos do curso de medicina, como, por exemplo, dissecções venosas, passagem de cateteres venosos e arteriais e passagem de dreno de tórax. Segundo Shimizu et al. (2002), pode-se destacar que nas unidades de internação são realizados alguns procedimentos de maior complexidade quando os pacientes correm risco de vida, o que aumenta a chance de a categoria médica se acidentar ${ }^{13}$. Além disso, se levarmos em consideração que esses jovens profissionais estão em processo de capacitação e adquirindo experiência dia a dia, espera-se que, com o decorrer de sua trajetória profissional, se exponham menos a acidentes.

As frequências de acidentes encontradas em "Acadêmicos de Medicina" são preocupantes. Esses dados corroboram o es- 
tudo de Reis et al. (2004), que relataram este fato e ainda acrescentaram que há um aumento no relato de acidentes à medida que eles avançam no curso de medicina, tendo como pico de notificação o décimo semestre de sua faculdade ${ }^{14}$. De acordo com um estudo internacional realizado na Universidade da Califórnia por Osborn et al. (1999), mais da metade dos estudantes de medicina, durante sua faculdade, envolveram-se em algum tipo de acidente com material biológico ${ }^{15}$. Em outro estudo, que incluiu 61 relatos de acidentes de trabalho no Hospital Universitário São Francisco de Paula, em Pelotas - RS, realizado por Monteiro et al. (2001), verificaram que 22,9\% dos acidentes com pérfuro-cortantes envolveram estudantes de medicina ${ }^{16}$.

Estes dados são muito importantes, pois denotam que, ao avançar no curso de medicina, os estudantes - que deveriam estar consolidando seu conhecimento em biossegurança e aplicando-os na prática do dia a dia para evitar exposições a materiais biológicos -, estão acidentando-se mais à medida que os procedimentos que realizam vão ficando mais complexos, situação esta que é alarmante e evidencia ser necessário rever as estratégias de educação em biossegurança desses profissionais em formação.

As categorias de acadêmicos, estagiários e profissionais em treinamento devem receber treinamentos adequados nestes cursos de formação técnica ou profissional. Esses treinamentos devem incluir, no mínimo, formas básicas de prevenção de acidentes e aplicação prática da Biossegurança, senão esses profissionais estarão sujeitos, certamente, a uma maior probabilidade de exposição a fatores nocivos, podendo culminar em graves acidentes ${ }^{17}$.

Podemos ressaltar em nosso estudo que a faixa etária mais acometida por acidentes com materiais biológicos foi a de 20-29 anos, sendo esta unanimidade entre as categorias profissionais investigadas. Oliveira et al. (2003) citam que a iniciação aos estágios e à pesquisa na vida acadêmica é um passo importante na busca de novas descobertas e, para isso acontecer, o acadêmico deve dispor de tempo hábil para poder se dedicar às suas responsabilidades de estagiário e/ou pesquisador. Com isso, os acadêmicos acabam tornando-se as pessoas que mais tempo permanecem expostas aos riscos de acidentes gerados em laboratórios de pesquisa. Caso tais instituições não disponham de treinamentos, palestras ou cursos em biossegurança, o aluno poderá colocar em risco sua saúde e a de seus colegas de trabalho ${ }^{18}$.

Segundo Carvalho et al. (1999), as pessoas mais suscetíveis a sofrerem acidentes ou malefícios à saúde são acadêmicos e estagiários jovens que não estão recebendo treinamento adequado ou atenção devida no que diz respeito às práticas de biossegurança executadas ${ }^{19}$.
Cosiglieri et al. (2002) evidenciaram que os acadêmicos geralmente não estão conscientizados nem familiarizados a respeito das medidas de segurança no ambiente de trabalho, quando deveriam receber treinamento continuado em disciplinas específicas para estes fins como, por exemplo, a biossegurança ${ }^{20}$.

Em nosso estudo não foi analisado o fator tempo de residência e período que os acadêmicos estariam cursando. Todavia, Costa et al.(1996) descreveram brilhantemente a relação tempo de trabalho e acidentes, ou seja, o número de acidentes é maior nos indivíduos que: 1) trabalham há pouco tempo em um ambiente de trabalho específico, devido à falta de experiência; 2) e nos indivíduos que trabalham nesse ambiente há muitos anos, devido ao excesso de confiança ${ }^{21}$. Oliveira et al. (2003) também mencionam que indivíduos com experiência, muitas vezes, se desconcentram e, por descuido próprio, cometem acidentes. Esta característica reforça a importância do desenvolvimento contínuo de treinamento, mesmo daqueles com mais experiência. Somente a partir de registros dos relatos de acidentes é que poderão ser formuladas e implementadas providências concretas, como treinamentos periódicos antes de iniciarem suas funções extracurriculares ou curriculares, e durante a permanência deste acadêmico no local de trabalho para que estes executem suas atividades com segurança ${ }^{18}$.

Costa et al. (1996) relataram ainda que a estatística dos acidentes de trabalho em saúde, assim como a grande maioria dos acidentes em geral, mostra maior frequência de casos em trabalhadores que apresentam excesso de confiança, e menor frequência de casos em trabalhadores que apresentam equilíbrio emocional e profissional ${ }^{21}$. Portanto, torna-se necessária a reciclagem sobre precauções universais e trabalho contínuo de educação, visando à prevenção de acidentes.

Quando observamos a frequência de notificações de acordo com o trimestre anual em que ocorreram os acidentes, constatamos que houve uma homogeneidade nas notificações nas três categorias, mantendo-se, em sua maioria, estáveis nos três primeiros trimestres e decaindo no último trimestre de cada ano. Sugere-se que este decréscimo deva estar relacionado ao ganho de experiência pelo acadêmico, que, a partir do desenvolvimento de suas habilidades nas atividades práticas durante o ano, tende a reduzir sua exposição a situações de risco.

Entretanto cabe ressaltar que um viés deste estudo são os casos de subnotificação. Este tipo de erro ocorre por diversos fatores. Uma investigação, com enfermeiros, sobre o tema demonstrou que $40 \%$ de eventos não foram relatados devido ao estigma da atitude negativa em relação ao incidente e os complexos relatórios que os profissionais devem realizar ${ }^{22}$. Outros 
autores identificaram o medo de represálias e exposição a julgamentos como os principais fatores para a não notificação e referem que apenas $25 \%$ dos erros são formalizados em relatórios ou notificações de ocorrências ${ }^{23}$. A subnotificação também está vinculada ao desconhecimento do que seja efetivamente um erro de medicação, além do desconhecimento frente às intercorrências que possam ocorrer com o paciente e do temor quanto a seu futuro profissional ${ }^{24,25}$.

Ao analisarmos o sítio anatômico mais acometido por acidentes, verificamos uma grande exposição dos membros superiores, geralmente por contusão relacionada a objetos pérfuro-cortantes, mais especificamente agulhas, sendo esta situação descrita com grande atenção por diversos autores ${ }^{26}$, 27, 28 , e pelo Center of Disease Control - CDC USA ${ }^{29}$, evidenciando uma grande preocupação já há mais de vinte anos e preconizando recomendações universais para prevenção dos acidentes causados por agulhas e também no que se refere à notificação dos acidentes e monitorização dos infectados, bem como à adoção de medidas de precauções-padrão pelos trabalhadores da saúde, visando à prevenção tanto da transmissão do vírus HIV quanto das Hepatites B e C.

Uma das principais causas dos acidentes de trabalho apontada por Sarquis et al. (1999) em acadêmicos e profissionais da saúde é a manipulação indevida de material pérfuro-cortante ${ }^{30}$, dados estes que fortalecem os achados de nosso estudo.

É evidente, em nosso estudo, que a exposição percutânea predominou sobre todos os outros tipos de exposição e que, apesar da presença de equipamentos de proteção individual nos locais de trabalho, ainda há acidentes envolvendo a pele e mucosas, o que está evidenciado já em dados obtidos por outros autores ${ }^{13,31,32}$

Os dados mostrados em nosso estudo trazem grandes preocupações com a categoria dos "Médicos Residentes" uma vez que estes são responsáveis pela maior porcentagem de acidentes envolvendo materiais biológicos provenientes de pacientes fonte com sorologia positiva para HIV ou HCV. A sorologia dos pacientes fonte envolvidos nos acidentes ocupacionais nos sujeitos de nosso estudo evidenciou um alto risco de contaminação, uma vez que, além dos "Médicos Residentes", os "Estudantes de Medicina" e os "Estagiários Técnicos de Enfermagem" foram fortemente afetados, merecendo um enfoque especial em seus currículos abordando temas relacionados à "Biossegurança", como a prevenção de acidentes, uso de equipamentos de proteção individual e a importância do relato imediato dos acidentes com materiais biológicos.

Dentre as causas mais relatadas pelos acadêmicos nos acidentes, a principal descrita foi o descuido próprio, que já foi citado em outros trabalhos na literatura como um dos principais fatores de predisposição a acidentes com materiais biológicos $^{33,34}$. Este fato deve ser cuidadosamente analisado para se traçar estratégias de ação de prevenção destes acidentes.

Baseando-se nessas evidências, propõe-se uma atenção especial à formação desses profissionais ao longo do curso desenvolvido. É necessário ressaltar que há problemas relacionados ao ensino da temática "Biossegurança", tanto em cursos superiores como nos técnicos e nas instituições de saúde. Verifica-se, ainda, que o ensino dessa temática nos cursos de nível técnico e graduação ocorrem de forma não sistematizada e descontínua, o que resulta na não aplicação desses conhecimentos quando os alunos se inserem no campo profissional ${ }^{35}$.

Tais resultados apontam para a necessidade de uma discussão aprofundada entre as instituições formadoras de profissionais da saúde, para que possa se encontrar estratégias de ensino mais eficientes para o desenvolvimento da temática da "Biossegurança", a fim de minimizar o número de acidentes com pérfuro-cortantes e materiais biológicos.

\section{CONCLUSÃO}

Acreditamos que é essencial implementar programas sistematizados para discutir biossegurança em todos os setores em que estão inseridos os médicos residentes, acadêmicos e estagiários das áreas da saúde, compreendendo estratégias eficazes para a prevenção e redução de risco de acidentes de trabalho, principalmente nos casos de exposição a material biológico. Além da permanente vigilância, faz-se necessário o incentivo da notificação de todo e qualquer acidente, pois assim será possível guiar a elaboração das medidas prevenção e, consequentemente, reduzir os riscos de acidentes.

A biossegurança é indiscutivelmente importante na formação tanto de profissionais da saúde quanto dos alunos graduação. Portanto, deve ser abordada no início dos cursos de graduação ou mesmo em cursos técnicos, pois o ingresso de profissionais mais conscientizados quanto à prevenção de acidentes e comportamentos seguros interfere diretamente no cenário atual de acidentes de trabalho, visto que eles protegem não só sua própria saúde e a de seus colegas, mas também a dos pacientes sob sua responsabilidade.

\section{CONSIDERAÇÕES FINAIS}

Este estudo forneceu dados de relevante pesquisa empírica, importantes para a revisão das estratégias de prevenção dos acidentes ocupacionais, mostrando a necessidade da intervenção nessa realidade.

Médicos residentes, acadêmicos e estagiários da área da saúde são inseridos diariamente em atividades relacionadas 
ao seu futuro profissional, muitas vezes sem experiência prévia, acabando por se exporem, desnecessariamente, a risco de acidentes de trabalho com materiais biológicos.

A prevenção através da educação continuada deve ser implementada ao longo dos cursos de graduação nas áreas da saúde, para garantir uma melhor postura dos profissionais que ingressam no mercado de trabalho, uma vez que o descuido próprio como a principal causa dos acidentes deve ser abolido.

Devem ser feitos futuros estudos que considerem as práticas educativas, mostrando aos alunos as condições concretas de vida e trabalho para que eles possam avançar na intervenção sobre a realidade social na qual agravos à saúde são produzidos.

\section{REFERÊNCIAS}

1. Risco Biologico.org [homepage]. [acesso em 28 abr. 2009]. Disponível em: http://www.riscobiologico.org/pagina_ basica.asp?id_pagina $=169$.

2. Binder MCP, Wludarski SL, Almeida IM. Estudo da evolução dos acidentes do trabalho registrados pela previdência social no período de 1995 a 1999, em Botucatu, SP. Cad Saúde Pública. 2001;17:915-24.

3. Cohn A, Karsh US, Hirano S, Sato AK. Acidentes do trabalho. Uma forma de violência. São Paulo: Brasiliense; 1985.

4. Ribeiro HP, Lacaz FAC. Acidentes de trabalho. De que adoecem e morrem os trabalhadores. São Paulo: Diesat; 1984.

5. Marziale MHP, Rodrigues CM. A produção científica sobre acidentes de trabalho com material perfurocortante entre trabalhadores de enfermagem. Rev Lat Amer Enferm. 2002;4:571-77.

6. Caixeta RB, Branco AB. Ocorrência de acidente do trabalho em uma unidade de terapia intensiva. Cad Saúde Pública. 2005;21(3):737-46.

7. Brasil. Ministério do Trabalho e Emprego. Portaria GM nº485, Segurança e Saúde no Trabalho em Estabelecimentos de Saúde. Diário Oficial da União. Brasília. 2005;131(94):6.

8. Guimarães RM, Mauro MYC. Potencial de morbimortalidade por acidente de trabalho no Brasil - período de 2002: uma análise epidemiológica. ALASS. 2004;55(2):18-20.

9. Pinho DLM, Rodrigues CM, Gomes GP. Perfil dos acidentes de trabalho no Hospital Universitário de Brasília. Rev Bras Enferm. 2007;60(3):29:1-4.

10. Nishide VM, Benatti MCC, Alexandre NMC. Ocorrência de acidente do trabalho em uma unidade de terapia intensiva. Rev Lat Amer Enferm. 2004;12(2):204-11.

11. Marcus VJB. Incidência de acidentes com materiais pérfuro-cortantes e fluidos corpóreos no hospital universitário “Alzira Velano" Alfenas. R Un Alfenas. 1999;5:221-5.
12. Cirolia LAS. Significado epidemiológico dos acidentes de trabalho com material biológico: hepatites B e C em profissionais da saúde. Rev Bras Med Trab. 2004;2(3):191-9.

13. Shimizu HE, Ribeiro EJG. Ocorrência de acidente de trabalho por materiais perfurocortantes e fluidos biológicos em estudantes e trabalhadores da saúde de um hospital escola de Brasília. Rev Esc Enferm USP. 2002;36(4):367-75.

14. Reis JMB. Acidentes relacionados ao treinamento durante atividade docente-discente-assistencial de estudantes de medicina. Rev Soc Bras Med Trop. 2004;37(5):405-8.

15. Osborn HE, Papadakis MA, Gerberding JL. Occupational exposures to body fluids among medical students. A 7-year longitudinal study. Ann of Intern Med. 1999;130:45-51.

16. Monteiro, PO. Perfil dos acidentes de trabalho no hospital Universitário São Francisco de Paula. In: IV Sul Encontro de Controle de Infecção. I Encontro de Microbiologia aplicada ao controle de infecção; 2001 jun. 27 - 30; Gramado, Brasil.

17. Júnior NM. Acidentes do trabalho em um hospital escola com instrumentos pérfuro-cortantes contaminados com material biológicos: o caso do Hospital Universitário da Universidade Federal de Santa Catarina. Florianópolis; 2003. Mestrado [Dissertação] — Universidade Federal de Santa Catarina.

18. Oliveira ESD. Acidentes gerados em Laboratórios de Pesquisa. Joinville; 2003. [Monografia] Curso de Graduação em Farmácia - Univille.

19. Carvalho PR. Boas Práticas Químicas em Biossegurança. Rio de Janeiro: Interciência; 1999.

20. Cosiglieri VO. Biossegurança em Laboratórios de ensino e da área da saúde. Manual de Biossegurança. São Paulo: Manole; 2002.

21. Costa MAF. Biossegurança: Química Básica em Biotecnologia e Ambientes Hospitalares. São Paulo: Santos; 1996.

22. Day G. Improving medication administration through an enhanced occurrence reporting system. J Nurs Care Qual. 1994;9(1):51-6.

23. Osborne J, Blais K, Hayes JS. Nurses's perceptions: when is it a medication error? J Nurs Adm. 1999;29(4):33-8.

24. Carvalho VT, Cassiani SHB. Análise dos comportamentos dos profissionais de enfermagem frente aos erros na administração de medicamentos. Acta Paul Enferm. 2002;15(2):45-54.

25. Bohomol E. Erros de Medicação: causas e fatores desencadeantes sob a ótica da equipe de enfermagem. São Paulo; 2002. Mestrado [Dissertação] — Universidade Federal de São Paulo.

26. Canini SEM. Situação de risco para transmissão de patógenos veiculados pelo sangue entre a equipe de enfermagem 
de um hospital universitário do interior paulista. São Paulo; 2000. Mestrado [Dissertação] — Escola de Enfermagem de Ribeirão Preto.

27. Brandi S, Benatti MCC, Alexandre NMC. Ocorrência de acidente de trabalho por material perfurocortante entre trabalhadores de enfermagem de um Hospital Universitário da Cidade de Campinas, Estado de São Paulo. Rev Esc Enferm USP. 1998;32:124-33.

28. Souza M. Acidentes ocupacionais e situações de risco para a equipe de enfermagem. São Paulo; 1999. Doutorado [Tese] — Escola Paulista de Medicina da Universidade Federal de São Paulo.

29. Center for Disease Control. Recommendations for prevention of HIV transmission in health-care settings. MMWR. 1988;36:3-17.

30. Sarquis LMM, Felli VEA. Acidentes de trabalho com instrumentos perfurocortantes entre os trabalhadores de enfermagem. Rev Esc Enferm USP. 2002;36(3):222-30.

31. Doebbeling BN. Protecting the healthcare worker from infection and injury. In: Wenzel RP, eds. Prevention and Control of Nosocomial Infections. Maryland: Williams \& Wilkins Company; 1997. p.397-402.

32. Queiroz MCB. Biossegurança. In: Oliveira AC, Albuquerque CP, Rocha LCM, eds. Infecções Hospitalares: Abordagem, Prevenção e Controle. Rio de Janeiro: Medsi; 1998. p.183-195.

33. Ribeiro MCP. Acidentes gerados em um laboratório de pesquisa. Joinville; 2003. [Monografia] - Univille.
34. Balsamo AC, Felli VEA. Estudo sobre os acidentes de trabalho com exposição aos líquidos corporais humanos em trabalhadores da saúde de um hospital universitário. Rev Lat Amer Enferm. 2006; 14(3):346-53.

35. Jansen AC. Um novo olhar para acidentes de trabalho: a questão do ensino. Ribeirão Preto; 1997. Mestrado [Dissertação] — Escola de Enfermagem de Ribeirão Preto.

\section{CONTRIBUIÇÃO DOS AUTORES}

Rafael Teixeira de Souza responsável pela coleta de dados, análise estatística, interpretação dos resultados e dissertação do artigo. Alana Durayski Ranzi contribuiu na coleta de dados e interpretação dos resultados. Carmem Susana Mondadori contribuiu na coleta de dados. Claudia Giuliano Bica orientou o projeto de pesquisa, contribuiu na interpretação dos resultados e dissertação do artigo

\section{CONFLITO DE INTERESSES}

Declarou não haver.

\section{ENDEREÇO PARA CORRESPONDÊNCIA}

Rafael Teixeira de Souza

Rua Sarmento Leite, 245

Bairro - Porto Alegre

CEP. 90050-170 RS

E-mail: rafafeevale@gmail.com 